\title{
The Routledge Handbook of Archaeological Human Remains and Legislation
}

\author{
- Edited by Nicholas Marquez Grant and Linda Fibiger •
}

Title: The Routledge Handbook of Archaeological Human Remains and Legislation: An International Guide to Laws and Practice in the Excavation and Treatment of Archaeological Human Remains

Editors: Nicholas Marquez Grant and Linda Fibiger

Edition: 1 st

Publisher: Routledge

Date of publishing: November 2010

Language: English

Format: Hardback

Size: $246 \times 174 \mathrm{~mm}$

Number of pages: 624

Number of illustrations: 25

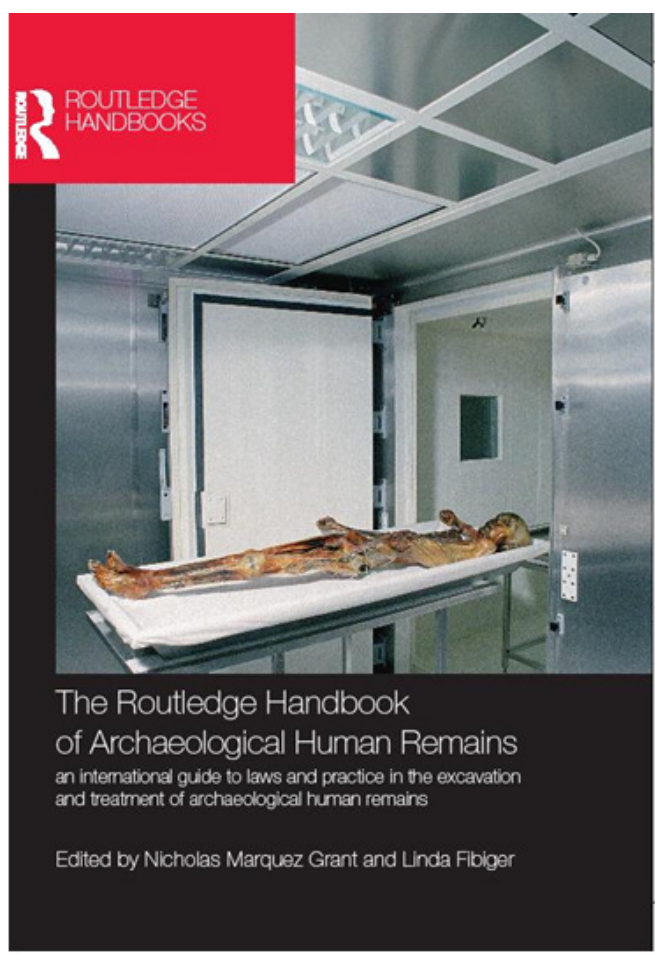

Price: $£ 150.00$

ISBN-13: 978-0-415-58857-7

Bull Int Assoc Paleodont. 2010;4(1):36-37.

The Routledge Handbook of Archaeological Human Remains and Legislation provides comprehensive information on the excavation of archaeological human remains and the law through sixty-two individual country contributions from Europe, Asia, Africa, North America, South America and Australasia. The volume addresses the following questions:

- What is the current situation (including a brief history, education and training) of physical anthropology in the country?

- What happens on discovering human remains (who is notified, what is the protocol)? 
- Where is the cut-off point between forensic and archaeological human remains (e.g. 100 years, 50 years, 25 years)?

- What is the current legislation regarding the excavation of archaeological human skeletal remains? Is a license needed to excavate human remains? Is there any specific legislation regarding the excavation of human remains, transportation, study and reburial? Any specific legislation regarding human remains from churchyards and those from recent war graves?

- Are physical anthropologists involved in the excavation process?

- What methods of anthropological analysis are mostly used in the country? Are there any population specific methods created in the country?

- Are their particular ethical issues that need to be considered when excavating human remains, such as religious groups or tribal groups?

The Routledge Handbook of Archaeological Human Remains and Legislation is an indispensible tool for archaeologists, anthropologists and other scientists involved in the excavation and study of human remains around the world.

For more information or to order the book please visit www.routledge.com/9780415588577

\section{Routledge \\ 兽 Taylor \& Francis Group}

